\title{
Importance of Graphical User Interface in the design of P300 based Brain-Computer Interface systems
}

Ratcliffe, Liam; Puthusserypady, Sadasivan

Published in:

Computers in Biology and Medicine

Link to article, DOI:

10.1016/j.compbiomed.2019.103599

Publication date:

2020

Document Version

Peer reviewed version

Link back to DTU Orbit

Citation (APA):

Ratcliffe, L., \& Puthusserypady, S. (2020). Importance of Graphical User Interface in the design of P300 based Brain-Computer Interface systems. Computers in Biology and Medicine, 117, [103599].

https://doi.org/10.1016/j.compbiomed.2019.103599

\section{General rights}

Copyright and moral rights for the publications made accessible in the public portal are retained by the authors and/or other copyright owners and it is a condition of accessing publications that users recognise and abide by the legal requirements associated with these rights.

- Users may download and print one copy of any publication from the public portal for the purpose of private study or research.

- You may not further distribute the material or use it for any profit-making activity or commercial gain

- You may freely distribute the URL identifying the publication in the public portal 


\title{
Importance of Graphical User Interface in the Design of P300 Based Brain-Computer Interface Systems
}

\author{
Liam Ratcliffe ${ }^{\mathrm{a}}$, Sadasivan Puthusserypady ${ }^{\mathrm{b}}$ \\ ${ }^{a}$ University of Glasgow, University Avenue, Glasgow G12 8QQ, United Kingdom. \\ ${ }^{b}$ Department of Health Technology, Technical University of Denmark, 2800 Kgs. Lyngby, Denmark.
}

\begin{abstract}
Objectives: Develop an effective and intuitive Graphical User Interface (GUI) for a Brain-Computer Interface (BCI) system, that achieves high classification accuracy and Information Transfer Rates (ITRs), while using a simple classification technique. Objectives also include the development of an output device, that is capable of real time execution of the selected commands. Methods: A region based T9 BCI system with familiar face presentation cues capable of eliciting strong P300 responses was developed. Electroencephalogram (EEG) signals were collected from the $\mathrm{Oz}, \mathrm{POz}$, $\mathrm{CPz}$ and $\mathrm{Cz}$ electrode locations on the scalp and subsequently filtered, averaged and used to extract two features. These feature sets were classified using the Nearest Neighbour Approach (NNA). To complement the developed BCI system, a 'drone prototype' capable of simulating six different movements, each over a range of eight distinct selectable distances, was also developed. This was achieved through the construction of a body with 4 movable legs, capable of tilting the main body forward, backward, up and down, as well as a pointer capable of turning left and right. Results: From ten participants, with normal or corrected to normal vision, an average accuracy of $91.3 \pm 4.8 \%$ and an ITR of $2.2 \pm 1.1$ commands/minute ( $12.2 \pm 6.0 \mathrm{bits} /$ minute) was achieved. Conclusion: The proposed system was shown to elicit strong P300 responses. When compared to similar P300 BCI systems, which utilise a variety of more complex classifiers, competitive accuracy and ITR results were achieved, implying the superiority of the proposed GUI. Significance: This study supports the hypothesis that more research, time and care should be taken when developing GUIs for BCI systems.
\end{abstract}

Keywords: Graphical User Interface (GUI), P300 speller, Brain-Computer Interface (BCI), Electroencephalogram (EEG), Event Related Potential (ERP), Nearest Neighbour Approach (NNA). 


\section{Introduction}

Brain-Computer Interface (BCI) systems are the implementation of an artificial communication link between an individual's brain and an external device via the processing and classification of detectable brain signals, such as the electroencephalogram (EEG) signals [1] 2]. BCI systems are presented throughout the literature as viable approaches to improving the quality of life of people suffering from neuromuscular diseases $[3]$. Farwell and Donchin [5] were the first to develop a BCI speller, a device capable of aiding in communication for those suffering from neuromuscular disorders. It utilised P300 Event Related Potentials (ERPs) which express time-locked relations with their stimuli [6]. The most robust feature of the P300 ERP is a large positive peak located around 300ms following stimulus presentation [7. These ERPs are shown to be highly stable responses that require no initial training to produce, allowing P300 based BCI systems to achieve reasonably high Information Transfer Rates (ITRs) [2]. Repeated P300 stimulation can be used to find an averaged response to a particular cue presentation. Averaging is used to improve the Signal-to-Noise Ratio (SNR) of a system [8] by minimising the background noise in the recording, keeping only the time-locked P300 signal.

The performance of BCI systems, the speed at which they can output information and aid with tasks, are measured by calculating their accuracies and ITRs [9]. There are two defining characteristics of a BCI system that determine its performance: the Graphical User Interface (GUI) and the classifier (the mathematical method(s) used to categorise incoming brain signals for command selection) 9]. The majority of BCI research publications focus predominantly on the development of the system's classifier [9], with competitions being held aiming to increase the classification accuracy of a set of offline data through the use of classifier variation and development alone [10. This paper aims to enhance the performance of a BCI system by modifying and optimising the GUI. The impact of the developed GUI is illustrated by combining it with a simple classification technique, Nearest Neighbour Approach (NNA) [1], and comparing it with existing BCI systems, which employ more complex classifiers. Using this, and the competitive accuracies and ITRs achieved, the effectiveness of the developed GUI is inferred. 


\section{State-of-the Art Review on GUIs}

Throughout the BCI literature, there exists numerous modifications to the original GUI developed by Farwell and Donchin in [5]. For example, Pan et al. [12] and Fazel-Rexai and Abhari [13, both in their respective publications have showed an increase in spelling accuracy for a region based speller when compared to a single display speller as in [5. Furthermore, Miao et al. [14] compared two GUIs and reported that the peripheral matrix paradigm achieved comparable results to the conventional matrix paradigm. Lu et al.15] analysed the effects of stimulus duration and inter-stimulus-interval (ISI) on BCI accuracy and ITR. They reported that a stimulus duration of $31.25 \mathrm{~ms}$ coupled with an ISI of $125 \mathrm{~ms}$ was the best compromise between elicited P300 signal strength and time taken to produce the response.

People's reactions to face stimuli vary considerably from other stimuli, and neuroimaging has identified occipito-temporal areas in humans that produce a much stronger response to stimuli consisting of faces [16]. Kaufman et al. [17] made use of this when designing their GUI and showed that using facial based cues increased both the induced magnitude of the ERP as well as the overall performance of the speller, due to the resulting higher SNR. Further, two publications by Li et al. [18] 19] compared the evoked ERPs of three spelling paradigms; the standard white square stimulus, a familiar face stimulus and a green familiar face stimulus. These works showed that the standard paradigm was out-preformed by the familiar face paradigm which was in turn out-preformed by the green familiar face paradigm. Inverted facial image cues have been used in another study and have displayed promising results for visual stimulus based BCIs [20].

Acqualagna et al. 21] utilised six different colours to stimulate six rows of the interface matrix. Another T9 GUI similar to that in [22] was developed by Ron-Angevin et al 23]. To mitigate the problems with double flashes and proximity-distraction, Postelnicu and Talaba 24] employed a checkerboard paradigm. A gaze-independent GUI, flashing groups on letters on the screen one after another, was developed in 25]. Pires et al. [26] showed the potential of a Gaze-Independent Block Speller (GIBS), placing letters in four blocks located in the screen corners, bringing them into the centre for further selection. The same authors proposed a lateral single-character speller to increase the spacing between letters reducing user distractions [27. Treder et al. 28] experimented with three, two-stage Hex-O-Spell variations. Aqualagna and Blankertz [29] also proposed a gazeindependent system by using Rapid Serial Visual Presentation (RSVP). In 2016, Yin et al. 30. developed a saccade-independent auditory and tactile P300 BCI system aimed at improving gaze 
shift-independent BCIs. Table 1 provides a summary of the range of BCI GUI's that are available in the literature to-date.

Table 1: Summary of the range of different GUIs found in the literature for BCI applications.

\begin{tabular}{|c|c|c|c|c|c|}
\hline $\begin{array}{l}\text { Speller } \\
\text { Type }\end{array}$ & Year & $\begin{array}{c}\text { \# of } \\
\text { channels }\end{array}$ & $\begin{array}{c}\text { \# of } \\
\text { subjects }\end{array}$ & $\begin{array}{c}\text { Accuracy } \\
(\%)\end{array}$ & $\begin{array}{c}\text { Speed } \\
\text { (bits/min) }\end{array}$ \\
\hline Region-based Spellers 12 & 2013 & 8 & 12 & 93.47 & 22.2 \\
\hline 13 & 2008 & 5 & 10 & 92 & - \\
\hline 14 & 2019 & 16 & 18 & 83.5 & 39.0 \\
\hline Stimulus Timings Variation [15] & 2013 & 32 & 6 & - & - \\
\hline Face Stimuli [17] & 2011 & 12 & 21 & up to 100 & - \\
\hline [18 & 2015 & 14 & 17 & - & - \\
\hline 19 & 2015 & 14 & 17 & 96.7 & 48.2 \\
\hline 20 & 2012 & 16 & 7 & 88.7 & 38.7 \\
\hline Chroma Speller 21 & 2013 & 64 & 9 & 88.4 & 11.2 \\
\hline T9 23 & 2015 & 8 & 11 & 93.75 & 13.9 \\
\hline 22 & 2015 & 8 & 10 & - & 26.7 \\
\hline Checkerboard Paradigm 24] & 2013 & 4 & 10 & 90.625 & 21.74 \\
\hline Giospell 25] & 2011 & 6 & 8 & $94.4-96.3$ & 11.0 \\
\hline Gaze-Independent Block 26] & 2011 & 12 & 4 & 96.02 & 16.67 \\
\hline Lateral Single-Character 27 & 2012 & 12 & 24 & 89.9 & 26.11 \\
\hline Hex-O-Spell with ERP 28 & 2011 & 64 & 13 & 91.3 & 9.8 \\
\hline RSVP 29 & 2013 & 63 & 12 & 94.8 & 11.4 \\
\hline Auditory and Tactile 20] & 2016 & 8 & 12 & 88.7 & 10.8 \\
\hline Proposed work & 2019 & 4 & 10 & 91.3 & 12.2 \\
\hline
\end{tabular}

All of the works listed in Table 1 aim to optimise BCI performance through modifications of parameters related to the GUI styling. In our work, we modify a range of parameters to optimise the GUI so that the elicited P300 responses are easily detectable.

\subsection{Take-Aways}

Several modifications to the original GUI developed by Farwell and Donchin in [5] are suggested in this work. First was the change in the output type, from letters used for communication to directional movements used for locomotion. The developed GUI has a total of 50 outputs. These include six directional commands; 'up', 'down', 'left', 'right', 'forward', and 'backward', plus the two commands for 'pause' and 'repeat'. Each of the directional commands has eight distance/speed variations. The developed GUI subsequently allows for the control of a drone prototype over both short and long distances, using small, accurate movements and gross, continual movements, respectively. It is anticipated that in the future, possible integration of the design into a wheelchair 
could enable disabled/elderly users to control and utilise the system both for short (indoor), as well as long (outdoor), navigation - a protocol that has been well documented in the literature [31, 32, 33, 34.

The second modification was the integration of a two stage interface each consisting of eight regions according to the suggestions by Pan et al. [12] and Fazal-Rexai and Abhari [13. Here, the stage one interface (interface 1) was designed to select movement direction, while stage two interface (interface 2) was designed to select the distance to be moved in the previously selected direction. These movements were either in metres or degrees depending on the selection in interface 1. The interfaces were arranged in a text on 9 keys (T9) layout, similar to the keypad of an old mobile phone [22]. As only eight options were available on each interface, the centre of the interface was left blank, providing space capable of displaying live user feedback via video in potential future developments [14].

Finally, as a third modification, a psychologically salient image of Albert Einstein was used as a stimulus, justified by [17, [18] and [19].

\section{Proposed GUI}

The proposed interface was developed with two sub-interfaces, each with different selection options. Interface 1, as shown in Fig. 1, allows the user to select a directional command. Upon selection of a command, one of the four different outcomes (listed below) can occur:

1. The 'repeat' command is selected, and interface 1 is shown again for another selection. The previously selected movement continues,

2. The 'up', 'down', 'forward' or 'backward' command is selected and variation 1 of the interface 2 (Fig. 2 ) is displayed for the next selection. Variation 1 allows for the selection of distance/speed control in the direction selected from interface 1,

3. The 'left' or 'right' command is selected and variation 2 of interface 2 is displayed for the next selection. Variation 2 allows for the selection of rotational distances/speeds in the direction already selected in interface 1 , ranging from $22.5^{\circ}$ to $180^{\circ}$ in steps of $22.5^{\circ}$, or

4. The 'pause' command is selected and the system pauses. Pressing the space bar restarts the system, returning to interface 1 . Following both variations of interface 2 , the system returns to interface 1 allowing for the next directional selection. 
Figure 1: A screen shot of interface 1 mid-cycle. It is seen that the current stimulated command is 'repeat'.

A snap-shot of interface 2, following an 'up' selection from interface 1 (Fig. 1), can be seen in Fig. 2,

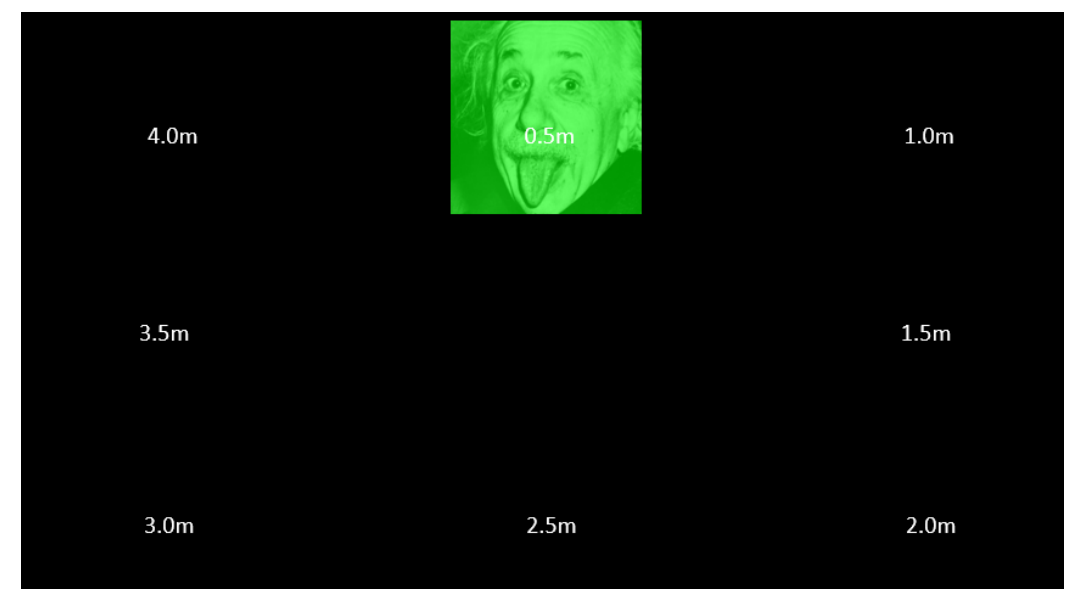

Figure 2: A screen shot of interface 2, variation 1. Currently the stimulated command is ' $0.5 \mathrm{~m}$ ' which, if selected, represents a translational movement of 0.5 meters in the direction selected in interface 1.

As the system takes the same time to select each new command, unless the 'repeat' or 'pause' command is selected, the outputs of distance also represent variations in speed. As a movement of $4 \mathrm{~m}$ and a movement of $0.5 \mathrm{~m}$ both occur over the same time frame, the speed of the $4 \mathrm{~m}$ movement is eight times greater than the movement of the $0.5 \mathrm{~m}$ movement. These are represented in the drone model accordingly. Figure 3 depicts a flow graph of all possible selections and the resulting next 
interface each selection prompts.

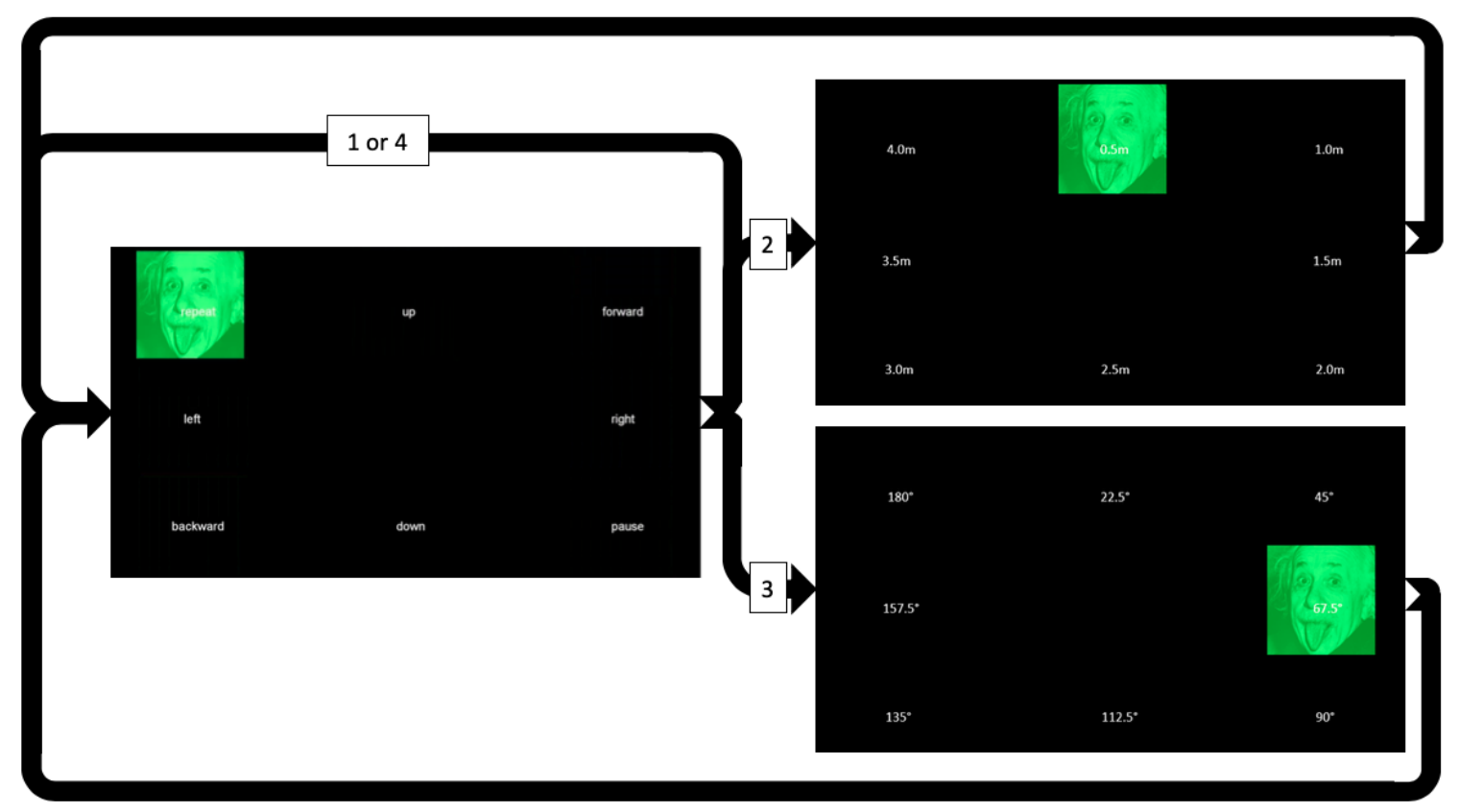

Figure 3: A flow graph of the progression of the GUIs following certain selections made. Following the list above; 1 - 'repeat' command is selected and interface 1 is shown again; 2 - 'up', 'down', 'forwards' or 'backwards' command is selected and variation 1 of interface 2 is displayed. Following a selection, interface 1 is displayed again; 3 - the 'left' or 'right' command is selected and variation 2 of interface 2 is displayed. Following a selection, interface 1 is displayed again; 4 - the 'pause' command is selected (here interface 1 is only displayed following the pressing of the space bar).

\section{Materials and Methods}

\subsection{Data Collection and Experimental Paradigm}

The GUI was displayed on the 15.6-inch FHD (1920 x 1080) anti-glare LED backlight IPS display of the Dell Inspiron 15 and was coded using the MATLAB extension, Psychtoolbox. The interface was split into eight regions, which flashed one at a time in a randomised order. Each flash lasted $31.25 \mathrm{~ms}$ and was separated from the next flash by a delay of $93.75 \mathrm{~ms}$, resulting in an ISI of $125 \mathrm{~ms}$. Eight flashes were needed to illuminate each region once, resulting in a total time of one second. Multiplying this by the number of times each region flashed per trial (denoted as $R$ ), gave the total time needed to make a selection. For example, when $R=10$, the total time taken for a selection was 10 seconds. 
All participants in the study were provided with written informed consent prior to their participation in the experiment, which were approved by the Regional Committee on Health Research Ethics for the Capital Region of Denmark (reference H-3-2013-004) and carried out in accordance with the corresponding guidelines and relevant regulations on the use of human participants for health-related scientific research. A post experiment questionnaire was also presented for participants to fill out upon completion of the trials.

Ten healthy participants ( $24 \pm 3$ years old, six males and four females) with normal or corrected to normal vision took part in the experiments. For each participant, two initial training trials were conducted to calibrate the system and classifier for distinguishing between two states: the brain's responses to a visual stimulus, and no stimulus. The EEG signals were recorded at a sampling rate of $256 \mathrm{~Hz}$ using four active surface electrodes placed above the parieto-occipital region of the brain at the $\mathrm{Oz}, \mathrm{POz}, \mathrm{CPz}$ and $\mathrm{Cz}$ electrode locations, according to the international 10-20 system [35. These 4 channels were selected following the unsuccessful initial testing of the system with only one channel $(\mathrm{Pz})$. The ground and reference electrodes were placed at Fpz and the left earlobe, respectively. The electrodes used were from the g.GAMMAbundle of the g.tec pack, used in conjunction with Nuprep skin prep gel, alcohol wipes and Signa electrode gel. The g.USBamp amplifier was used to collect the data for further processing.

During the experiment, the participant was sat on a chair $60 \mathrm{~cm}$ away from the computer screen, with the top of the GUI at the participant's eye level. Instructions for the task were given ahead of the experiment. For the first run, the value of $R$ was set to 10. This resulted in 80 flashes per trial, ten for each of the eight regions of the GUI, lasting 10 seconds in total. Care was taken to make sure that no region was flashed twice in succession and that the flash order was randomised each time.

Each set of trials involved 34 selection phases consisting of two calibration phases and 32 online selection phases. At the start of each set of trials, two calibration trials were performed, where the participant was instructed to concentrate on the 'up' command for both trials. These two trials were averaged to detect the participant's average response to both stimulus and no stimulus. The next 32 trials were then split into four sets of eight trials, so that each of the eight commands - 'repeat', 'up', 'forward', 'left', 'right', 'backwards', 'down', and 'pause' were selected four times. Between each of these trials, a 4.1 second period of relaxation was provided for selection feedback, blinking and adjusting gaze for the next command. This 4.1 seconds period consists of two components: (i) 
1.1 seconds for a buffer to allow for the last 1 second of EEG data to enter the classifier following the last cue presentation, and (ii) 3 seconds to display the chosen command to the participant and allow them some time to blink as well as to prepare for the next selection. It should also be noted that by reducing this time, say to 2.1 seconds, an increase in the corresponding ITR would be achieved. After the first eight trials, 60 seconds break was given to the participant to rest. This was repeated four times. From this, an accuracy was calculated, and the value of $R$ was adjusted to find the best compromise between speed and accuracy for a particular participant. That is, its value was decreased if the participant's accuracy was above $90 \%$, speeding up the system but reducing accuracy, and increased if the participant's accuracy was below $90 \%$, slowing down the system but increasing the accuracy. For each participant, the lowest value of $R$ that maintained an accuracy of over $90 \%$ was used in the final results.

\subsection{Signal Processing}

The signals from the electrode locations $\mathrm{Oz}, \mathrm{POz}, \mathrm{CPz}$ and $\mathrm{Cz}$ were filtered using a 4th-order Butterworth $50 \mathrm{~Hz}$ notch filter to minimise the power-line interference followed by a $0.1 \mathrm{~Hz}$ 4th-order Butterworth high pass filter and a $30 \mathrm{~Hz}$ 4th-order Butterworth low pass filter to reduce the out of band noise. The responses to cue presentation for each of the eight regions were then averaged, resulting in eight averaged responses corresponding to eight regions. Each of these eight averaged responses in turn consisted of four sets of data, one for each of the four electrode locations. An example response from participant 1 is illustrated in Fig. 4.

Signals from these four channels were then combined for each region using the following formula:

$$
O z_{i}+P O z_{i}-C P z_{i}-C z_{i}
$$

where, $i$ is the sample index. Eq. (1) was derived (empirically) from our observations of the recorded signals during the development. As can be seen in Fig. 4. 0.5 seconds after the cue presentation, variations in signal (averaged) amplitudes were fairly consistent for all four channels over time. The only significant separation of the signal amplitudes was seen in an interval of about $0.2-0.3$ seconds after cue presentation. Figure 5 shows the result of combining (using Eq.(1D) the four responses seen in Fig. 4 From this signal, two features were extracted, the location and value of the maximum amplitude of the resulting signal. This can be seen in Fig. 5 where the only region of significant separation occurs again between 0.2 and 0.3 seconds following cue presentation. Similar plots were 


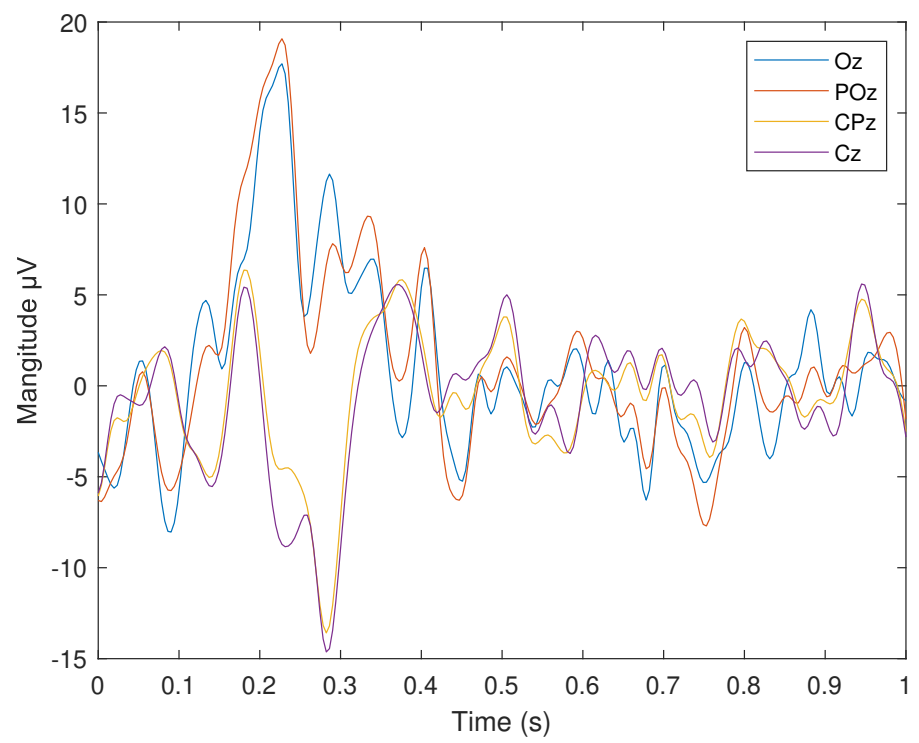

Figure 4: Example of one second (averaged) responses from participant 1 at four electrode locations following the presentation of the stimulus.

found for all eight regions for each trial and used for NNA, comparing each of these feature pairs with that of the average from the two trial runs.

\subsection{Nearest Neighbour Approach (NNA)}

It is one of the simplest and best-known classification algorithms [11. For a given test pattern, the NNA assigns the class label of its nearest neighbour.

Here, we have eight training patterns corresponding to eight regions (classes). Let us denote them as, $\left(\mathbf{x}_{i}, c_{i}\right), i=1,2, \ldots, 8$, where $\mathbf{x}_{i}=\left(f_{1}^{i}, f_{2}^{i}\right)$ are the two features corresponding to the $i^{\text {th }}$ region/class $\left(c_{i}\right)$. Let us also denote the target feature pair as $\mathbf{T}_{\mathbf{f}}=\left(t f_{1}, t f_{2}\right)$. The NNA then estimates $D_{i}=\left\|\mathbf{x}_{i}-\mathbf{T}_{\mathbf{f}}\right\|, i=1,2, \ldots, 8$, the Euclidean distance between $\mathbf{T}_{\mathbf{f}}$ and $\mathbf{x}_{i}$ and chooses the class $\left(c_{i}\right)$ which has the lowest distance estimate.

An example scatter plot containing all 34 trials is provided in Fig. 6. Here, the two red crosses are the feature pairs from the training trials for participant 1 , which were averaged and used as the target for NNA $\left(t f_{1}\right.$ and $\left.t f_{2}\right)$. The blue crosses show the resulting feature pairs acquired from the recorded brain signals following cue presentation within the region of interest. Finally, the green crosses represent the resulting feature pairs from the recorded brain signals following cue 


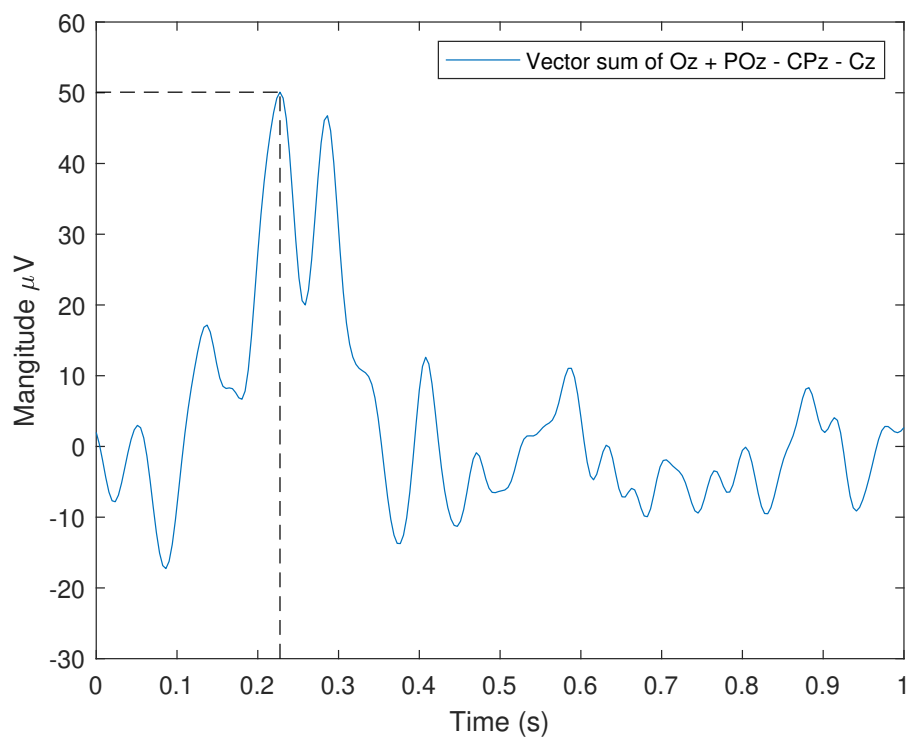

Figure 5: Result of performing the operation in Eq. (1) to the signals in Fig. 4 Two dotted lines show the values of feature 1 and feature 2. This feature set was one of the two calibration trials used for participant 1.

presentation at the other seven regions (regions of non-interest). Note here that some of the blue crosses are located far away from the training sets. This is a result in errors in eliciting the P300 response resulting in a $<100 \%$ classification accuracy.

Figure 7 illustrates the feature pair scatter plots for all the ten participants. The caption states the corresponding values of $R$ used for each participant. These values are consistent with those used in the results section of this paper.

\subsection{Information Transfer Rate (ITR)}

As explained in [36], the optimal measure of a communication system's performance is its ITR, accounting for both number of selections and the accuracy of these selections [37. Due to the two stage GUI developed in this work, every selection error resulted in the additional two selections before a successful command selection. Therefore, as in 38, a practical ITR has been used to better estimate the speed of communication. The practical ITR (in bits per minute) was calculated using the following equations [37] 38]

$$
\begin{aligned}
B & =\log _{2} N+P \log _{2} P+(1-P) \log _{2}\left(\frac{1-P}{N-1}\right), \\
I T R_{\text {Bits }} & =\frac{B}{T},
\end{aligned}
$$




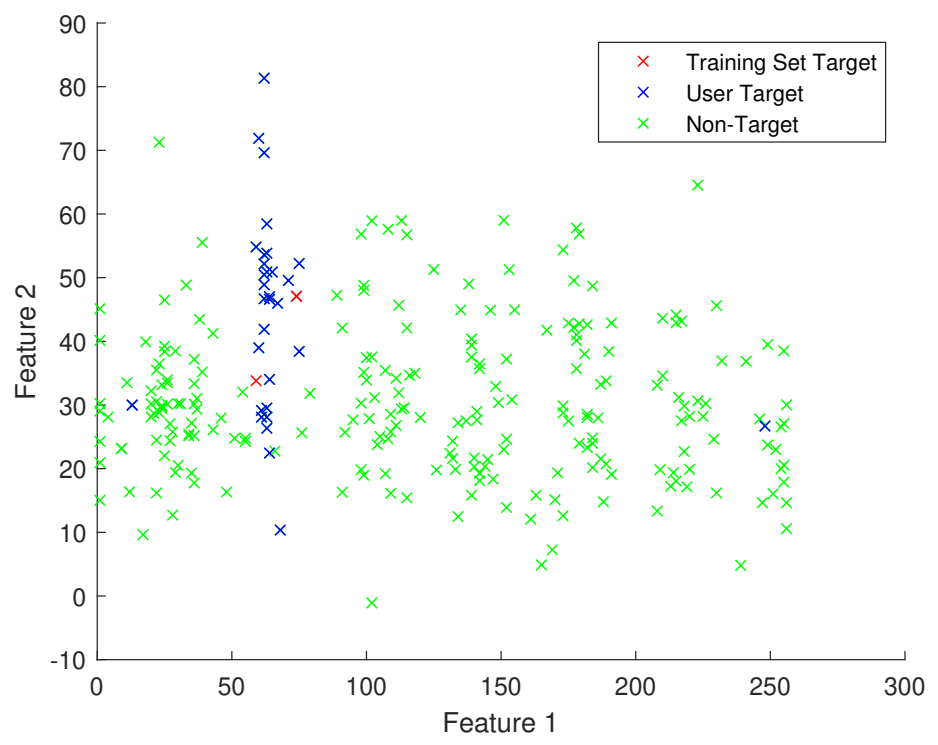

Figure 6: The resulting feature scatter plot from participant 1 over three averaged stimuli $(R=3)$, showing two 'Training Set Targets' which were averaged for $\mathbf{T}_{\mathbf{f}}, 32$ 'User Targets' depicting the brains response to stimulus presentation, and 224 'Non-Targets' depicting the brains response to no stimulus presentation.

where, $B$ is the number of bits transmitted per trial, $N(=50)$ is the number of possible selections, $P$ is the classification accuracy for that user and $T$ is the average selection time in minutes and is calculated using the following formula:

$$
T=\frac{2 T_{1}+6 T_{2}}{8 \times 60}
$$

Here, $T_{1}$ and $T_{2}$ are the two selection times corresponding to the selections made using interface 1 alone and both interfaces, respectively. $T_{1}=R+4.1$ and $T_{2}=2 T_{1}=2 R+8.2$, where 4.1 and 8.2 are the times needed for selection feedback. Assuming random selection, $T_{1}$ ('repeat' and 'pause' commands) is used as the selection time $2 / 8$ times.

A second measure of ITR is in commands per minute. To calculate it for each participant, first it is required to find the total number of selections $(S)$ each participant would need in order to successfully make the $N(=50)$ selections. According to [38, $S$ is given by,

$$
S=\frac{N}{1-2 P}
$$

The product $\left(\frac{S}{N}\right) T$ is the average time required to select a correct command and hence the com- 


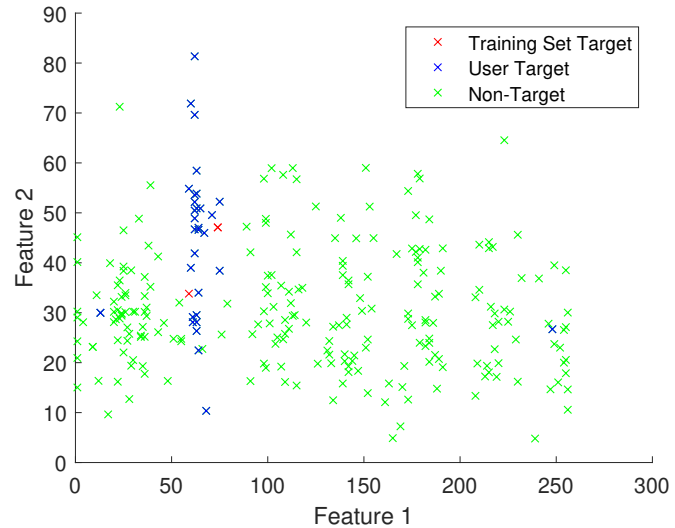

(a) Participant \#1

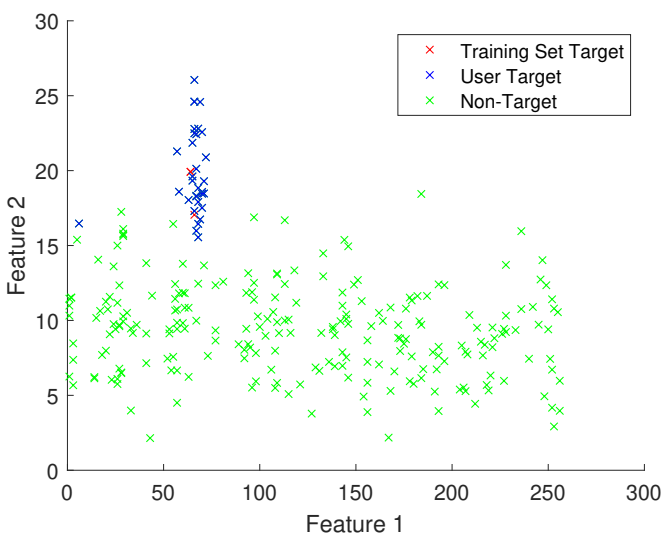

(c) Participant \#3

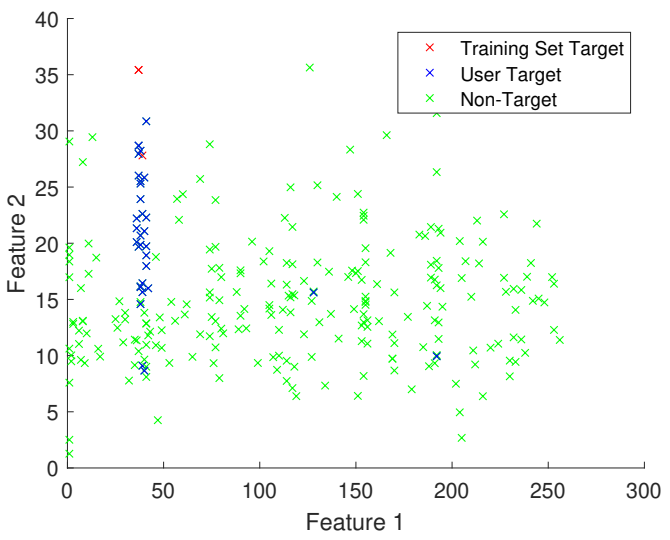

(e) Participant \#5

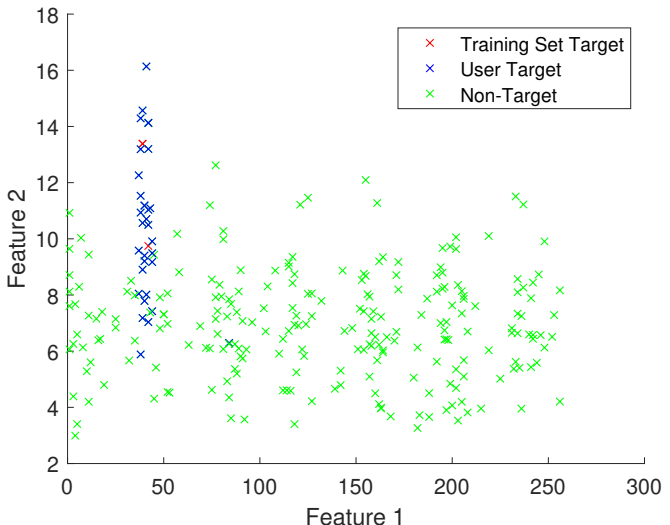

(b) Participant \#2

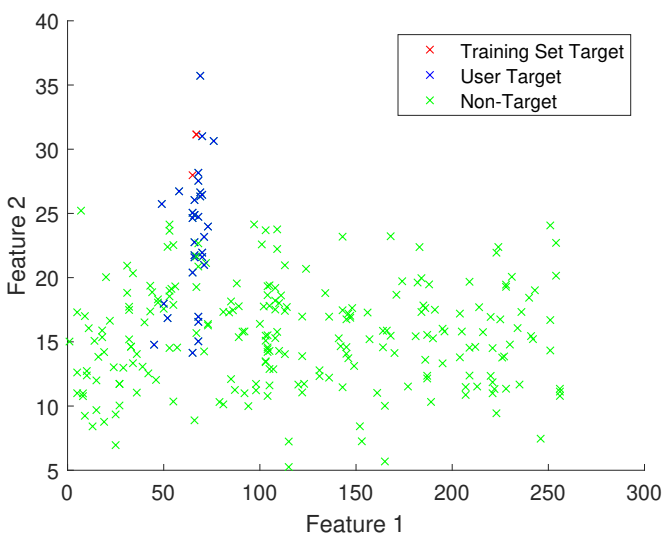

(d) Participant \#4

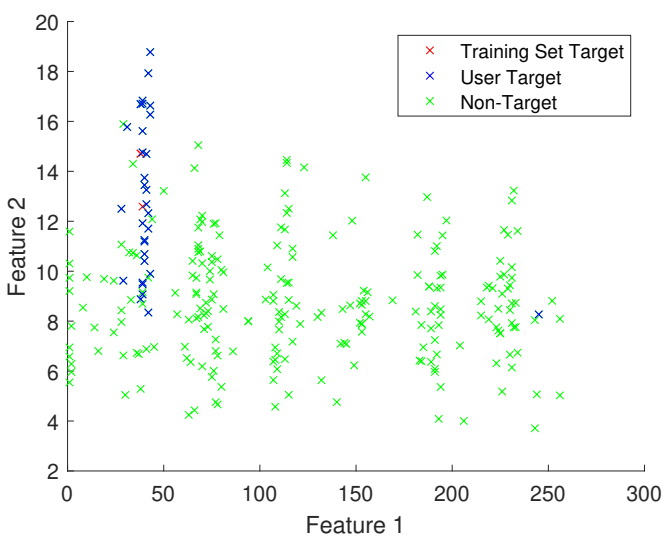

(f) Participant \#6 


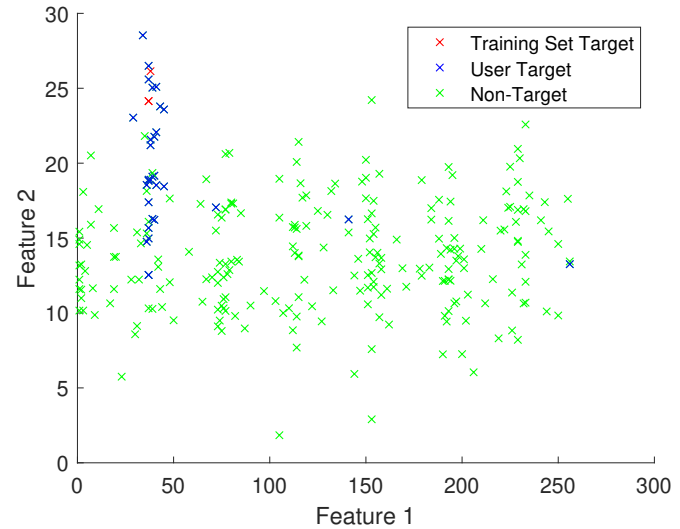

(g) Participant \#7

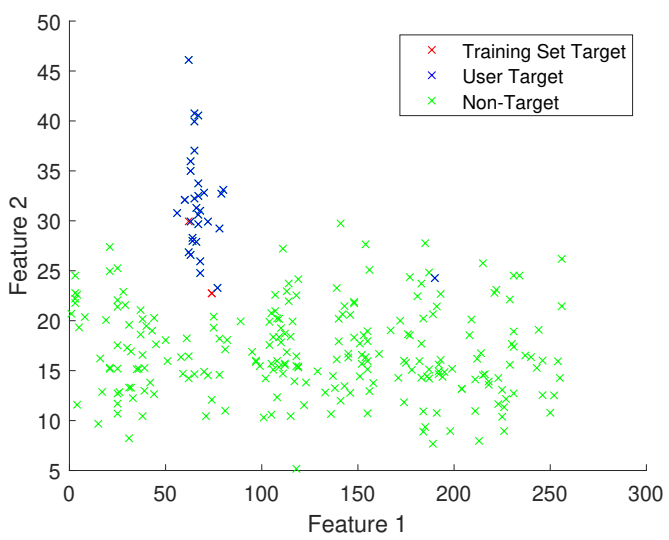

(i) Participant \#9

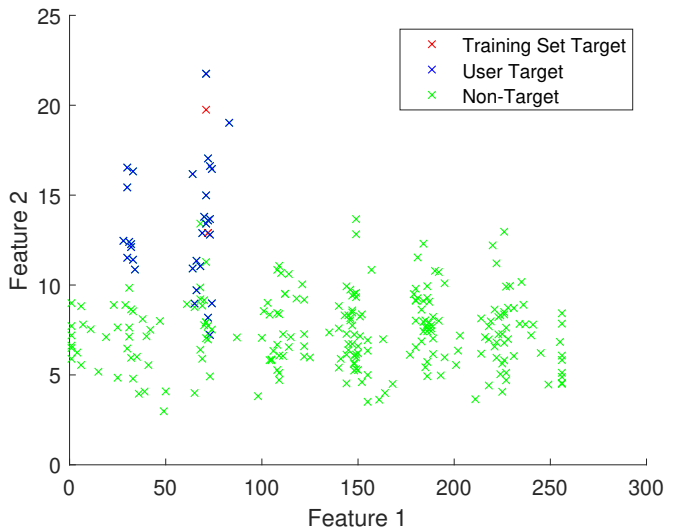

(h) Participant \#8

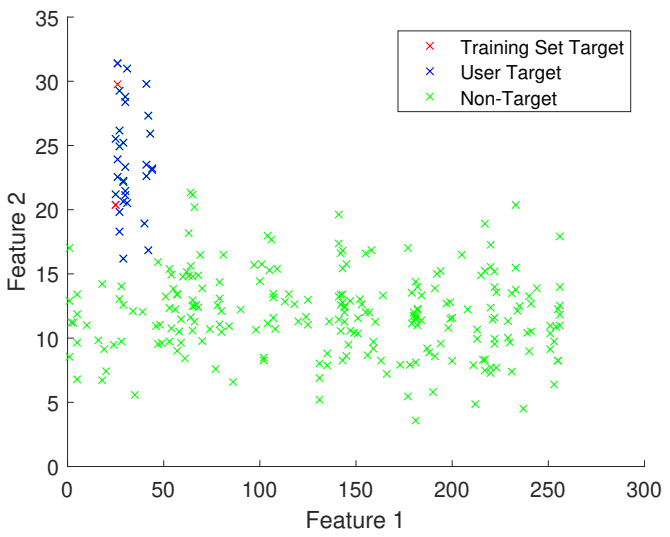

(j) Participant \#10

Figure 7: Feature pair scatter plots of all 10 participants, as a reflection of the results displayed in Table 2 These results attained the highest ITR for each participant while maintaining a sufficiently high accuracy: (a) $R=3$, (b) $R=15$, (c) $R=12$, (d) $R=10$, (e) $R=5$, (f) $R=20$, (g) $R=15$, (h) $R=20$, (i) $R=6$, (j) $R=12$. 
mands per minute can be calculated as,

$$
I T R_{\text {Commands }}=\frac{60}{\frac{S}{N} T}=\frac{60 N}{S T}
$$

\section{Results and Discussion}

\subsection{Classification Accuracies and ITRs for the Proposed System}

The classification accuracies and ITRs of the online simulation for all ten participants are reported in Table 2. The P300 classification remained highly accurate for all participants, barring participant 8 , with a mean accuracy of $91.3 \pm 4.8 \%$. Due to the varying values of $R$ used by each participant, the resulting ITR rates varied significantly, with an average speed of $2.2 \pm 1.1$ commands per minute / $12.2 \pm 6.0$ bits per minute.

The value of $R$ varied (between 3 and 20) largely between participants. Its value was limited, on the low end, when the accuracy for the participant fell below $90 \%$, and on the high end, by the value of 20. Anything higher was deemed too lengthy for sufficient maintenance of concentration. These two extremes can be seen in the results obtained by participant $1(R=3)$ and participant 8 $(R=20)$.

Table 2: Online classification accuracies and ITRs for the developed P300 speller.

\begin{tabular}{cccc}
\hline $\begin{array}{c}\text { Participant } \\
\text { No. }\end{array}$ & $\begin{array}{c}\text { Accuracy } \\
(\%)\end{array}$ & $\begin{array}{c}\text { ITR } \\
\text { (commands/minute) }\end{array}$ & $\begin{array}{c}\text { ITR } \\
\text { (bits/minute) }\end{array}$ \\
\hline 1 & 93.8 & 4.3 & 24.0 \\
2 & 90.6 & 1.5 & 8.4 \\
3 & 96.6 & 2.0 & 11.2 \\
4 & 90.6 & 2.0 & 11.3 \\
5 & 93.6 & 3.3 & 18.6 \\
6 & 87.5 & 1.1 & 6.3 \\
7 & 87.5 & 1.4 & 7.9 \\
8 & 81.3 & 1.0 & 5.5 \\
9 & 96.9 & 3.2 & 17.9 \\
10 & 93.8 & 1.9 & 10.6 \\
\hline Average & 91.3 & 2.2 & 12.2 \\
Std & 4.8 & 1.1 & 6.0 \\
\hline
\end{tabular}

Figure 8 shows a scatter plot of the mean accuracies and ITRs from all of the comparable P300based spellers discussed in [9]. A least-squares regression line was added to illustrate the trend of these results. It also depicts the universal trade-off between increasing ITR and the resulting 
decrease in accuracy of the system. The average results from this paper, represented by the red cross, falls above the line of least squares, suggesting competitive accuracy and ITR among existing P300 BCI systems of recent years. A summary of all the articles used in our comparison can be seen in Table 3 ,

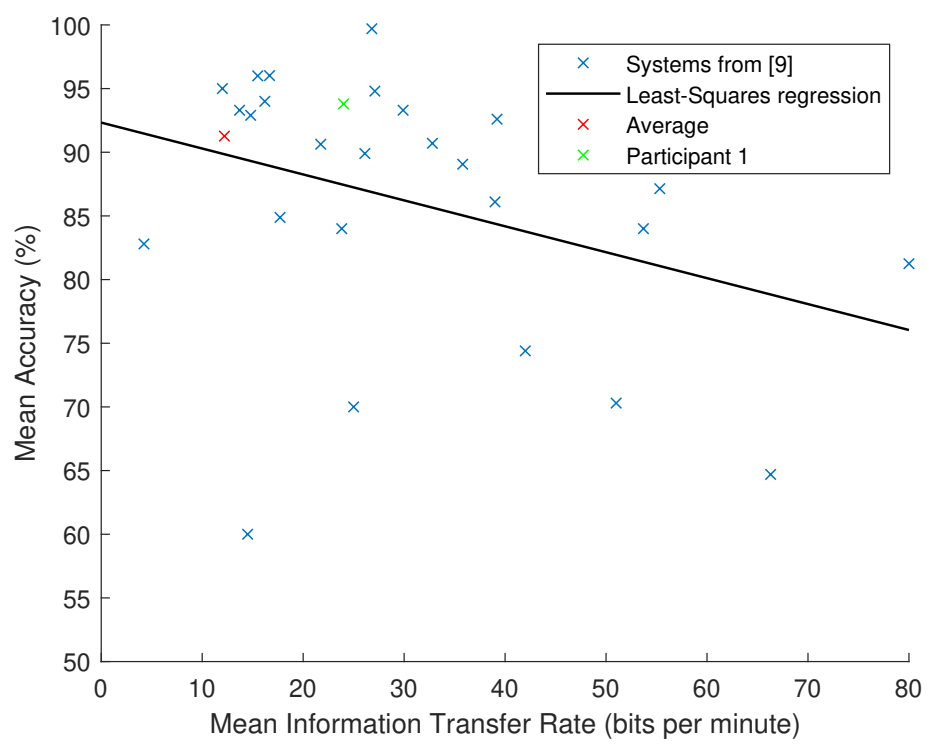

Figure 8: A scatter plot of the mean accuracies and mean ITRs from the P300 spellers discussed in 9 , with the addition of the average results and the results achieved by participant 1 from this paper. The black line in the graph represents the least-squares regression line.

The results from participant 1, the best performing participant, are also included on the graph (green cross). It is hypothesised that with the addition of a more complex classifier, similar results could be achieved for all participants, further improving the performance of the developed BCI. Such classifiers include Shrinkage Linear Discriminant Analysis (SLDA) [57, sparse Bayesian [58, Spatial-Temporal Discriminant Analysis (STDA) [59] and Step-Wise Linear Discriminant Analysis (SWLDA). These are variations of LDA classifiers which are used to maximise the separability among the known categories. Looking at the the NNA plots in Fig. 7 separation of the two categories could be vastly improved through the use of higher dimensionality analysis coupled with better features for separation. This would maintain separation between the two categories for lower values of $R$ for each user, increasing the ITR of the system. These improvements are predicted to be largest for participants that achieved lower ITRs in the NNA results. 
Table 3: A summary of all the comparable P300-based spellers ITRs and accuracies used in Fig. 8

\begin{tabular}{|c|c|c|c|}
\hline $\begin{array}{l}\text { Speller } \\
\text { Type }\end{array}$ & Reference & $\begin{array}{c}\text { Mean ITR } \\
\text { (bits/min) }\end{array}$ & $\begin{array}{c}\text { Mean Accuracy } \\
(\%)\end{array}$ \\
\hline Matrix & 5 & 12.0 & 95.0 \\
\hline \multirow{6}{*}{ Stimuli Variation } & [40] & 66.3 & 64.7 \\
\hline & 41 & 13.7 & 93.3 \\
\hline & 42 , & 35.8 & 89.06 \\
\hline & 43 & 26.8 & 99.7 \\
\hline & 44 & 14.8 & 92.9 \\
\hline & 45 & 23.82 & 84.0 \\
\hline \multirow{4}{*}{ Familiar Faces and Symbols } & [18] & 39.0 & 86.1 \\
\hline & [46] & $\sim 80$ & 81.25 \\
\hline & 47 & $32.8-53.7$ & $84.0-90.7$ \\
\hline & [48] & $15.5-16.2$ & $94-96$ \\
\hline \multirow{2}{*}{ Variation of Letters Arrangement } & [49] & 55.32 & 87.14 \\
\hline & [50] & $27.1-29.9$ & $93.3-94.8$ \\
\hline \multirow{2}{*}{ Matrix Speller with Prediction } & 51 & 17.71 & 84.88 \\
\hline & [52] & 25 & 70 \\
\hline \multirow{3}{*}{ Other Languages } & 53 & 14.5 & 60 \\
\hline & 54 & 4.23 & 82.8 \\
\hline & 55 & 39.2 & 92.6 \\
\hline Checkerboard Paradigm & 24 & 21.74 & 90.63 \\
\hline Gibs & 26 & 16.67 & 96.02 \\
\hline LSC Speller & [27] & 26.11 & 89.9 \\
\hline RSVP & [56] & $42-51$ & $70.3-74.4$ \\
\hline Region T9 speller (familiar face cues) & Proposed work & 12.2 & 91.3 \\
\hline
\end{tabular}

\subsection{Drone Prototype}

To complement the BCI system, a drone prototype was made utilising an Arduino, several accessory components, Solidworks and a laser cutter. The prototype developed was used to display the BCIs ability for real time control of a device capable of movement in a $3 \mathrm{D}$ environment. It was designed to react in real time to commands selected by the user, and execute these commands while the next command selection was being made. A schematic of this setup can be seen in Fig. 9. The assembly of the set-up with the laser-cut components can be seen in Fig. 10.

The prototype was made with the aim to intuitively model the movements of an actual drone capable of six movements selectable within the interface. This was possible using the four legs as seen in Fig. 10 and Fig. 11. The 6050 MPU accelerometer was used to detect the tilt, velocity and acceleration of the main body. Forward and backward movements were simulated by the forward 


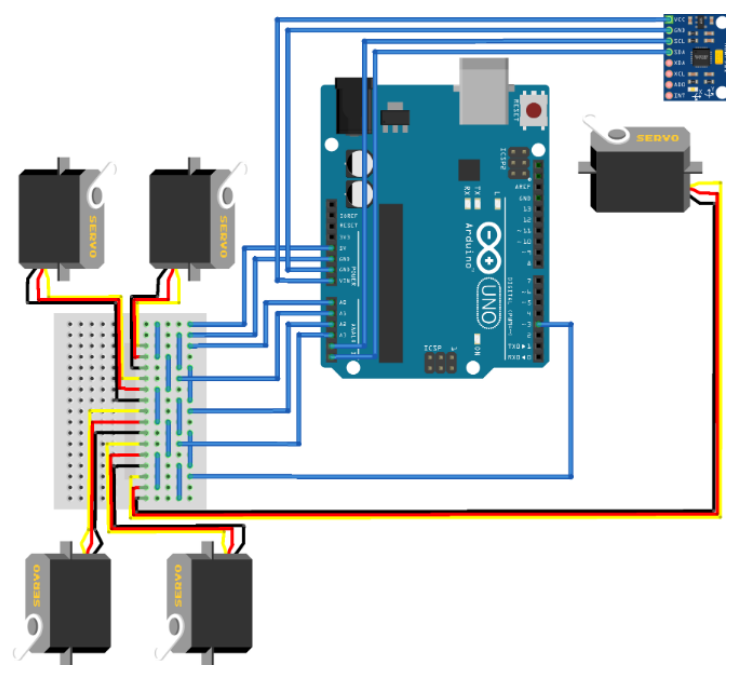

Figure 9: A schematic of the drone prototype.

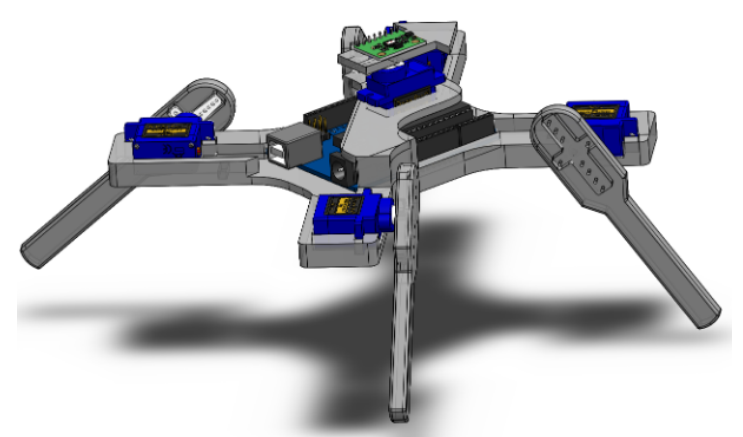

Figure 10: A screen caption of the assembly made using Solidworks. Consisting of the main body, four legs, one pointer, five servos [60], one mini breadboard [61, one $6050 \mathrm{MPU}$ 62] and one Arduino Uno 63. 
and backward tilting of the main body. This was achieved by increasing or decreasing the angles of the front two legs while decreasing or increasing the angles of the back two legs. In order to represent different speeds and distances selected by interface 2, different tilt angles were used. It was decided that larger tilt angles would be used to represent larger forward and backward movements. The amount of tilt on the main body was detected by the MPU. Up and down movements were simulated by the vertical movements of the prototype. Here, change in body height away from the resting state, represented the velocity in the upward or downward direction. Again, greater increases/decreases in height represented greater speeds in that direction. Finally, left and right movements were represented by the rotating pointer. Similar principles apply as up and down movements, with the angular displacement of the pointer representing the angular velocity of the prototype. An illustration of the final design can be seen in Fig. 11. This has been imaged in the resting position, representing a hovering drone with no translational or rotational movements. A full video of the developed P300 BCI system working alongside the prototype can be found in 1

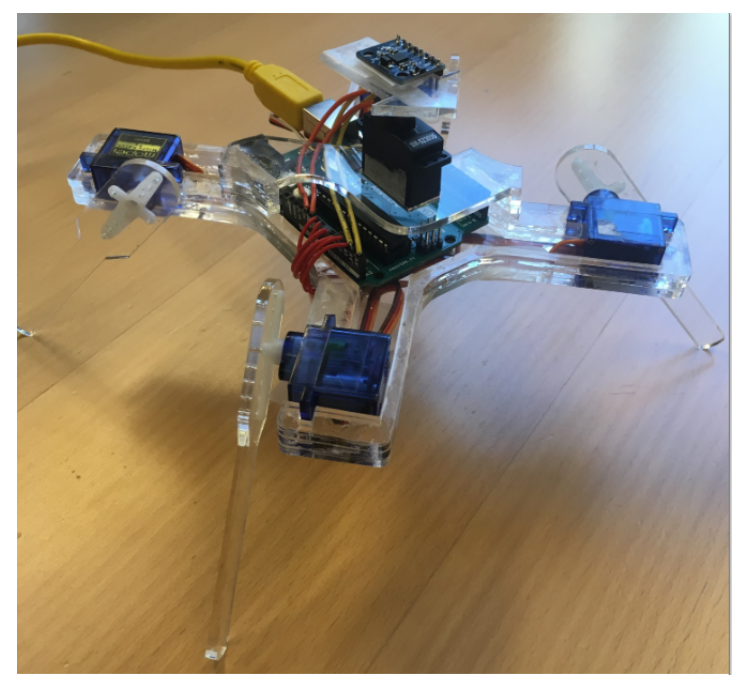

Figure 11: The final model used to simulate the drone movements for an online proof-of-concept for directional control.

\footnotetext{
${ }^{1}$ https://www.youtube.com/watch?v=cHSuR3ZGIIo
} 


\section{Conclusion}

In this work, the elicitation of sufficiently strong P300 ERPs was achieved using the proposed region based T9 GUI with familiar face presentation cues. These P300 responses have subsequently been classified using NNA and the system performance achieved a classification accuracy of $91.3 \pm 4.8 \%$ and an ITR of $2.2 \pm 1.1$ (commands/minute) / $12.2 \pm 6.0$ (bits/minute). The resulting competitive performance within the existing BCI field (see Fig. 8) further illustrates the GUI's ability to elicit strong and easily classifiable ERPs. Finally, the supporting material of the drone prototype illustrates the real time capabilities of the developed BCI, providing the proof-of-concept for future use in locomotive control for wheelchair users with only minor modifications to the GUI and the supporting code. This implementation, coupled with a more complex classifier, could prove highly beneficial for the potential users.

\subsection{Future Directions}

Three main areas of future development have been recognised following the completion of this study, which are listed below:

- Further comparison of the GUI with other BCI paradigms: Alterations to the detected EEG signals, such as Steady-State Visual Evoked Potentials (SSVEPs) or Motor Imagery (MI) signals, would allow for further GUI comparison. Modifications required for SSVEP-based BCI include: (i) the stimuli - a range of distinguishable frequencies to stimulate different regions of the GUI, and (ii) the classifier - features in the frequency domain for detection. For MI-based BCIs, the modifications include: (i) the interface - a selection feedback system to be included allowing users to concentrate on different bodily movements to select commands, (ii) the classifier - detecting MI induced signals from EEG, and (iii) training - users must be MI literate before using the device. Combinations of these two paradigms would also allow for further comparisons.

- Further improvements to the GUI styling: Brought about by testing altercations to any parameter of the existing GUI. Examples include: (i) using the inverse face presentation (visual stimuli) as in [20], (ii) varying the number of electrodes and their placements, (iii) implementation of ErrP detection (to detect the selection errors), and (iv) variations in stimuli locations and spacing. 
- Improvements to the BCI through classifier variation testing: Examples include SLDA, sparse Bayesian, STDA, and SWLDA.

Finally, additional online testing of the GUI alongside the developed drone prototype is needed to further the understanding of the GUI's real world capabilities.

\section{Acknowledgements}

The authors would like to thank the Hearing Systems Group of the Department of Health Technology, DTU, for providing the EEG facility for data collection. Special thanks to former PhD student Jakob Møller, for his help at the beginning of the project. Lastly, we express our sincere gratitude to all the participants who volunteered to participate in the experiments.

\section{References}

\section{References}

[1] J. Vidal, Toward direct brain computer communication, Annu. Rev. Biophys. Bioeng. 2 (1973) $157-180$ (1973).

[2] J. Wolpaw, N. Birbaumer, D. McFarland, G. Pfurtscheller, T. Vaughan, Brain computer interfaces for communication and control, Clin. Neurophysiol 113 (2002) 767-791 (2002).

[3] R. Carabalona, F. Grossi, A. Tessadri, P. Castiglioni, A. Caracciolo, et. al., Light on! real world evaluation of a P300-based brain-computer interface (BCI) for environment control in a smart home, Ergonomics 55 (2012) 552-563 (2012).

[4] S. Kleih, T. Kaufmann, C. Zickler, S. Halder, F. Leotta, F. Cincotti, F. Aloise, A. Riccio, C. Herbert, D. Mattia, A. Kübler, Out of the frying pan into the fire - - the P300-based BCI faces real-world challenges, Progress in Brain Research 194 (2011) 27-46 (2011).

[5] L. Farwell, E. Donchin, Talking off the top of your head: toward a mental prosthesis utilizing event related brain potentials, Clin. Neurophysiol. 70 (1988) 510-523 (1988).

[6] D. Ming, X. An, Y. Xi, Y. Hu, B. Wan, H. Qi, L. Cheng, Z. Xue, Time-locked and phaselocked features of P300 event-related potentials (ERPs) for brain computer interface speller, Biomedical Signal Processing and Control 5 (2010) 243-251 (2010). 
[7] N. Xu, X. Gao, B. Hong, X. Miao, S. Gao, F. Yang, BCI competition 2003 - data set iib: enhancing P300 wave detection using ICA-based subspace projections for BCI applications, IEEE Transactions on Biomedical Engineering 51 (2004) 1067-1072 (2004).

[8] M. Rugg, M. Coles, Electrophysiology of Mind: Event-Related Brain Potentials and Cognition, 1st Edition, Oxford University Press, 1995 (1995).

[9] A. Rezeika, M. Benda, P. Stawicki, F. Gembler, A. Saboor, I. Volosyak, Brain computer interface spellers: A review, Brain Sciences 8 (2018) 1-38 (2018).

[10] B. Blankertz, BCI competition III website, Available at http://http://www.bbci.de/ competition/iii/descII.pdf (2004).

[11] P. Clark, F. Evans, Distance to nearest neighbor as a measure of spatial relationships in populations, Ecology 35 (1954) 445-453 (1954).

[12] J. Pan, Y. Li, Z. Gu, Z. Yu, A comparison study of two P300 speller paradigms for brain computer interface, Cognitive Neurodynamics 7 (2013) 523-529 (2013).

[13] R. Fazel-Rezai, K. Abhari, A comparison between a matrix-based and a region-based P300 speller paradigms for brain-computer interface, 30th Annual International Conference of the IEEE Engineering in Medicine and Biology Society (2008) 1147-1150 (2008).

[14] Y. Miao1, E. Yin, B. Allison, Y. Zhang, Y. Chen, Y. Dong, X. Wang, D. Hu, A. Chchocki, J. Jin, An ERP-based BCI with peripheral stimuli: validation with ALS patients, Cognitive Neurodynamics (2019) 1-13 (2019).

[15] J. Lu, W. Speier, X. Hu, N. Pouratian, The effects of stimulus timing features on P300 speller performance, Clinical neurophysiology: official journal of the International Federation of Clinical Neurophysiology 124 (2013) 306-314 (2013).

[16] D. Tsao, S. Moeller, W. Freiwald, Comparing face patch systems in macaques and humans, PNAS 105 (2008) 19514-19519 (2008).

[17] T. Kaufmann, S. Schulz, C. Grnzinger, A. Kbler, Flashing characters with famous faces improves ERP-based brain computer interface performance, Journal of Neural Engineering 8 (2011) 1-10 (2011). 
[18] Q. Li, S. Liu, J. Li, Neural mechanism of P300-speller brain-computer interface using familiar face paradigm, IEEE 1 (2015) 611-614 (2015).

[19] Q. Li, S. Liu, J. Li, O. Bai, Use of a green familiar faces paradigm improves P300-speller brain-computer interface performance, PLoS One 10 (2015) e0130325 (2015).

[20] Y. Zhang, Q. Zhao, J. Jin, X. Wang, A. Cichocki, A novel BCI based on ERP components sensitive to configural processing of human faces, Neural Engineering 9 (2012) pp: 026018 (2012).

[21] L. Acqualagna, M. Treder, B. Blankertz, Chroma speller: Isotropic visual stimuli for truly gazeindependent spelling, 2013 6th International IEEE/EMBS Conference on Neural Engineering (NER) 6 (2013) 1041-1044 (2013).

[22] F. Akram, S. Han, T. Kim, An efficient word typing P300-BCI system using a modified T9 interface and random forest classifier, Computers in Biology and Medicine 65 (2015) 30-36 (2015).

[23] R. Ron-Angevin, S. Varona-Moya, L. Silva-Sauer, Initial test of a T9-like P300-based speller by an ALS patient, Journal of Neural Engineering 12 (2015) pp: 046023 (2015).

[24] C. Postelnicu, D. Talaba, P300-based brain-neuronal computer interaction for spelling applications, IEEE Transactions on Biomedical Engineering 60 (2013) 534-543 (2013).

[25] Y. Liu, Z. Zhou, D. Hu, Gaze independent brain-computer speller with covert visual search tasks, Clinical Neurophysiology 122 (2011) 1127-1136 (2011).

[26] G. Pires, U. Nunes, M. Castelo-Branco, GIBS block speller: Toward a gaze-independent P300based BCI, 2011 Annual International Conference of the IEEE Engineering in Medicine and Biology Society (2011) pp: 6360-6364 (2011).

[27] G. Pires, U. Nunes, M. Castelo-Branco, Comparison of a row-column speller vs. a novel lateral single-character speller: Assessment of BCI for severe motor disabled patients, Clinical Neurophysiology 123 (2012) 1168-1181 (2012).

[28] M. Treder, N. Schmidt, B. Blankertz, Gaze-independent brain-computer interfaces based on covert attention and feature attention, Journal of Neural Engineering 8 (2011) pp: 066003 (2011). 
[29] L. Acqualagna, B. Blankertz, Gaze-independent BCI-spelling using rapid serial visual presentation (RSVP), Clinical Neurophysiology 124 (2013) 901-908 (2013).

[30] E. Yin, T. Zeyl, R. Saab, Z. Zhou, T. Chau, An auditory-tactile visual saccade-independent P300 brain-computer interface., International Journal of Neural Systems 26 (2016) pp: 1650001 (2016).

[31] I. Iturrate, J. Antelis, A. Kubler, J. M. J, A noninvasive brain-actuated wheelchair based on a P300 neurophysiological protocol and automated navigation, IEEE Transactions on Robotics 25 (2009) 614-627 (2009).

[32] B. Rebsamen, C. Guan, H. Zhang, C. Wang, C. Teo, et. al., A brain controlled wheelchair to navigate in familiar environments, IEEE Transactions on Neural Systems and Rehabilitation Engineering 18 (2010) 590-598 (2010).

[33] G. Gentiletti, J. Gebhart, R. Acevedo, . Yáñez-Suárez, V. Medina-Bañuelos, Command of a simulated wheelchair on a virtual environment using a brain-computer interface, IRBM 30 (2009) 218-225 (2009).

[34] O. Piña-Ramirez, R. Valdes-Cristerna, O. Yanez-Suarez, Scenario screen: A dynamic and context dependent P300 stimulator screen aimed at wheelchair navigation control, Computational and Mathematical Methods in Medicine 10 (2018) 1-16 (2018).

[35] H. Jasper, The 10-20 electrode system of the international federation, Electroencephalography and Clinical Neurophysiology 10 (1954) 371-375 (1954).

[36] J. Shen, J. Liang, J. Shi, Y. Wang, A dynamic submatrix-based P300 online brain-computer interface, Biomedical Signal Processing and Control 15 (2015) 27-32 (2015).

[37] D. McFarland, W. Sarnacki, J. Wolpaw, Brain-computer interface (BCI) operation: optimizing information transfer rates, Biol. Psychol. 63 (2003) 237-251 (2003).

[38] G. Townsend, B. LaPallo, C. Boulay, D. Krusienski, G. Frye, C. Hauser, N. Schwartz, T. Vaughan, J. Wolpaw, E. Sellers, A novel P300-based brain-computer interface stimulus presentation paradigm: moving beyond rows and columns, Clin. Neurophysiol. 121 (2010) 1109-1120 (2010). 
[39] W. Speier, C. Arnold, N. Pouratain, Evaluating true BCI communication rate through mutual information and language models, PLoS ONE 8 (2013) e78432 (2013).

[40] S. Yeom, S. Fazli, S. Lee, P300 visual speller based on random set presentation, 2014 International Winter Workshop on Brain-Computer Interface (BCI) 1 (2014) 1-2 (2014).

[41] Q. Obeidat, T. Campbell, J. Kong, Introducing the edges paradigm: A P300 brain-computer interface for spelling written words, IEEE Transactions on Human-Machine Systems 45 (2015) $727-738$ (2015).

[42] Y. Liu, Z. Zhou, D. Hu, Comparison of stimulus types in visual P300 speller of brain-computer interfaces, 9th IEEE International Conference on Cognitive Informatics (ICCI'10) 9 (2010) 273-279 (2010).

[43] J. Shi, J. Shen, Y. Ji, F. Du, A submatrix-based P300 brain-computer interface stimulus presentation paradigm, Journal of Zhejiang University SCIENCE C 13 (2012) 452-459 (2012).

[44] J. Jin, P. Horki, C. Brunner, X. Wang, C. Neuper, et. al., A new P300 stimulus presentation pattern for EEG-based spelling systems, Biomedizinische Technik/Biomedical Engineering 55 (2010) 203-210 (2010).

[45] C. Polprasert, P. Kukieattikool, T. Demeechai, J. Ritcey, S. Siwamogsatham, New stimulation pattern design to improve P300-based matrix speller performance at high flash rate, Journal of Neural Engineering 10 (2013) pp: 036012 (2013).

[46] T. Kaufmann, A. Kübler, Beyond maximum speed-a novel two-stimulus paradigm for braincomputer interfaces based on event-related potentials (P300-BCI), Journal of Neural Engineering 1 (2014) pp: 056004 (2014).

[47] S. Yeom, S. Fazli, K. Müller, S. Lee, An efficient ERP-based brain-computer interface using random set presentation and face familiarity., PLoS ONE 9 (2014) pp: e111157 (2014).

[48] I. Käthner, A. Kübler, S. Halder, Rapid P300 brain-computer interface communication with a head-mounted display, Frontiers in Neuroscience 9 (2015) pp: 056004 (2015).

[49] S. Ahi, H. Kambara, Y. Koike, A dictionary-driven P300 speller with a modified interface, IEEE Transactions on Neural Systems and Rehabilitation Engineering 19 (2011) 6-14 (2011). 
[50] J. Jin, E. Sellers, X. Wang, Targeting an efficient target-to-target interval for P300 speller brain-computer interfaces, Medical \& Biological Engineering \& Computing 50 (2012) 289=296 (2012).

[51] D. Ryan, G. Frye, G. Townsend, D. Berry, S. Mesa-G, et. al., Predictive spelling with a P300based brain-computer interface: Increasing the rate of communication, International journal of human-computer interaction 27 (2011) 69 (2011).

[52] T. Kaufmann, S. Völker, L. Gunesch, A. Kübler, Spelling is just a click away - a user-centered brain-computer interface including auto-calibration and predictive text entry, Frontiers in Neuroscience 6 (2012).

[53] J. Minett, G. Peng, L. Zhou, H. Zheng, W. Wang, An assistive communication brain-computer interface for chinese text input, 2010 4th International Conference on Bioinformatics and Biomedical Engineering 4 (2010) 1-4 (2010).

[54] J. Minett, H. Zheng, M. Fong, L. Zhou, G. Peng, et. al., A chinese text input brain-computer interface based on the P300 speller, International Journal of Human-Computer Interaction 28 (2012) 472-483 (2012).

[55] Y. Yu, Z. Zhou, E. Yin, J. Jiang, Y. Liu, et. al., A P300-based brain-computer interface for chinese character input, International Journal of Human-Computer Interaction 32 (2016) 878-884 (2016).

[56] H. Sato, Y. Washizawa, An N100-P300 spelling brain-computer interface with detection of intentional control, Computers 5 (2016) 31 (2016).

[57] B. Blankertz, S. Lemm, M. Treder, S. Haufe, K. Müller, Single-trial analysis and classification of ERP components - a tutorial, NeuroImage 56 (2010) 814-825 (2010).

[58] Y. Zhanga, G. Zhou, J. Jin, Q. Zhao, X. Wang, A. Cichocki, Sparse bayesian classification of EEG for brain-computer interface, IEEE Transactions on Neural Networks and Learning Systems 27 (2016) 2256-2267 (2016).

[59] Y. Zhang, G. Zhou, Q. Zhao, J. Jin, X. Wang, A. Cichocki, Spatial-temporal discriminant analysis for ERP-based brain-computer interface, IEEE Transactions on Neural Systems and Rehabilitation Engineering 21 (2013) 233-243 (2013). 
[60] M. Frasson, Sg90 - micro servo 9g - tower pro, Available at https://grabcad.com/library/ sg90-micro-servo-9g-tower-pro-1 (2017).

[61] A. Aelevanthara, Mini breadboard, Available at https://grabcad.com/library/ mini-breadboard (2012).

[62] E. Rodriguez, Mpu $6050 \mathrm{imu}$ sensor, Available at https://grabcad.com/library/ mpu-6050-imu-sensor-1 (2015).

[63] L. Enrique, Arduino uno, Available at https://grabcad.com/library/arduino-uno-18 (2018). 